\title{
Incidence and predictors of dural venous sinus pressure gradient in idiopathic intracranial hypertension and non-idiopathic intracranial hypertension headache patients: results from 164 cerebral venograms
}

\author{
Michael R. Levitt, MD, ${ }^{1-3}$ Randall J. Hlubek, MD, ${ }^{4}$ Karam Moon, MD, ${ }^{4}$ \\ M. Yashar S. Kalani, MD, PhD, ${ }^{4}$ Peter Nakaji, MD, ${ }^{4}$ Kris A. Smith, MD, ${ }^{4}$ Andrew S. Little, MD, ${ }^{4}$ \\ Kerry Knievel, DO, ${ }^{5}$ Jane W. Chan, MD, ${ }^{6}$ Cameron G. McDougall, MD, ${ }^{4}$ and \\ Felipe C. Albuquerque, MD ${ }^{4}$
}

\begin{abstract}
Departments of ${ }^{1}$ Neurological Surgery, ${ }^{2}$ Radiology, and ${ }^{3}$ Mechanical Engineering, University of Washington, Seattle, Washington; and Departments of ${ }^{4}$ Neurosurgery, ${ }^{5}$ Neurology, and ${ }^{6}$ Neuro-Ophthalmology, Barrow Neurological Institute, St. Joseph's Hospital and Medical Center, Phoenix, Arizona
\end{abstract}

OBJECTIVE Cerebral venous pressure gradient (CVPG) from dural venous sinus stenosis is implicated in headache syndromes such as idiopathic intracranial hypertension $(\mathrm{IIH})$. The incidence of CVPG in headache patients has not been reported.

METHODS The authors reviewed all cerebral venograms with manometry performed for headache between January 2008 and May 2015. Patient demographics, headache etiology, intracranial pressure (ICP) measurements, and radiographic and manometric results were recorded. CVPG was defined as a difference $\geq 8 \mathrm{~mm} \mathrm{Hg}$ by venographic manometry.

RESULTS One hundred sixty-four venograms were performed in 155 patients. There were no procedural complications. Ninety-six procedures (58.5\%) were for patients with IIH. The overall incidence of CVPG was $25.6 \%$ (42 of 164 procedures): $35.4 \%$ (34 of 96 procedures) in IIH patients and $11.8 \%$ (8 of 68 procedures) in non-IIH patients. Sixty procedures $(36.6 \%)$ were performed in patients with preexisting shunts. Seventy-seven patients $(49.7 \%)$ had procedures preceded by an ICP measurement within 4 weeks of venography, and in $66(85.7 \%)$ of these patients, the ICP had been found to be elevated. CVPG was seen in $8.3 \%(n=5)$ of the procedures in the 60 patients with a preexisting shunt and in $0 \%$ ( $n$ $=0$ ) of the 11 procedures in the 77 patients with normal ICP ( $<<0.001$ for both). Noninvasive imaging (MR venography, CT venography) was assessed prior to venography in $112(68.3 \%)$ of 164 cases, and dural venous sinus abnormalities were demonstrated in $73(65.2 \%)$ of these cases; there was a trend toward CVPG ( $p=0.07)$. Multivariate analysis demonstrated an increased likelihood of CVPG in patients with IIH (OR 4.97, 95\% CI 1.71-14.47) and a decreased likelihood in patients with a preexisting shunt (OR 0.09, 95\% Cl 0.02-0.44).

CONCLUSIONS CVPG is uncommon in IIH patients, rare in those with preexisting shunts, and absent in those with normal ICP.

http://thejns.org/doi/abs/10.3171/2015.12.JNS152033

KEY WORDS benign intracranial hypertension; headache; idiopathic intracranial hypertension; manometry; pseudotumor cerebri; venography; interventional neurosurgery 
$\mathrm{C}$ EREBRAL venous hypertension has been implicated in the development of headache disorders such as idiopathic intracranial hypertension (IIH), and dural venous sinus stenting has emerged as an alternative to CSF shunting in such patients. ${ }^{2,15}$ Noninvasive imaging such as MR venography (MRV) and CT venography (CTV) may show anatomical stenosis or atresia of the venous sinuses in up to $93.1 \%$ of IIH patients and $48.9 \%$ of chronic daily headache patients but in only $6.8 \%$ of controls. ${ }^{9,11}$ However, cerebral venography with manometry is the gold standard for determining the cerebral venous pressure gradient (CVPG) across a dural venous sinus stenosis, and this procedure is used to determine which patients are likely to benefit from subsequent dural venous sinus stenting. ${ }^{1}$

The prevalence of, and the risk factors for, CVPG in the IIH and non-IIH headache populations are not well characterized. Such information could be used to determine which patients are most likely to benefit from venographic manometry and subsequent dural venous sinus stenting. We analyzed a large series of consecutive venograms obtained in both IIH and non-IIH headache patients to determine the incidence of CVPG, and developed a predictive model of CVPG risk factors.

\section{Methods \\ Patient Population}

This study was approved by the Institutional Review Board of St. Joseph's Hospital and Medical Center, Phoenix, Arizona. All cerebral venograms with manometry for the indication of headache that were performed at the institution between January 2008 and May 2015 were retrospectively reviewed. Patients were referred for venography and manometry by primary care physicians, neurologists, and neurosurgeons. Other than the diagnosis of headache, there were no specific inclusion criteria for referral, but patients with acute cerebral venous sinus thrombosis and arteriovenous malformations were excluded. In patients with IIH, diagnosis was made on the basis of symptoms referable to intracranial hypertension, intracranial pressure $(\mathrm{ICP}) \geq 25 \mathrm{~cm} \mathrm{H}_{2} \mathrm{O}$, normal CSF contents, and no structural cause of elevated ICP on neuroimaging. ${ }^{12} \mathrm{We}$ recorded patient demographic characteristics and body mass index (BMI; calculated as weight in $\mathrm{kg} /$ height in $\mathrm{m}^{2}$ ); headache diagnosis; presence of a preexisting CSF shunt; recent (within 30 days prior to venography) ICP measurement by lumbar puncture, ventricular catheterization, or ICP monitor; and recent (within 3 months prior to venography) noninvasive cerebrovascular imaging by brain MR or CT angiography or venography. Findings of noninvasive imaging were considered abnormal by independent neuroradiological interpretation if a focal stenosis or an atresia was seen.

\section{Venography Procedure}

Cerebral venography was performed under local anesthesia in all patients 18 years or older and under general anesthesia in all pediatric patients (age $<18$ years). Standard transfemoral venous access was obtained and a 5-Fr sheath was inserted. A 5-Fr angled catheter (Envoy MPD,
DePuy Synthes Co.) was placed over a Glidewire (Terumo Medical Corp.) and navigated into the right jugular bulb, unless significant stenosis necessitated placement in the left jugular bulb. A microcatheter (SL-10 or XT-17, Stryker Neurovascular) and 0.014-inch microwire (Synchro-2, Boston Scientific Corp.) were navigated into the dural venous sinuses. The microwire was removed, and a standard arterial pressure transducer was calibrated and connected to the microcatheter. Manometry was attempted in the following locations: anterior, middle, and posterior onethird of the superior sagittal sinus; bilateral transverse and sigmoid sinuses; and bilateral jugular bulbs. In patients with dural venous sinus compression from an extrasinus mass, manometry was performed only in the sinus segments proximal and distal to the mass. In patients in whom portions of the venous sinus system were absent, measurements were obtained in all other locations. Cerebral venography of the superior sagittal sinus and bilateral transverse and sigmoid sinuses was also performed through microcatheter injection. All catheters and the femoral sheath were then withdrawn, and hemostasis was obtained by mechanical pressure for 5 to 10 minutes, followed by 2 hours of flat bedrest. Manometry was considered positive when a CVPG of $8 \mathrm{~mm} \mathrm{Hg}$ or greater was found between any 2 anatomically contiguous segments of the dural venous sinuses (e.g., transverse and sigmoid sinuses). ${ }^{1}$

\section{Statistical Analysis}

Frequencies with percentages and standard deviations with means were used to describe the overall study cohort. Independent-samples t-tests were used to compare mean differences for continuous variables, and chi-square or Fisher exact tests were used for categorical variables. Four patient variables (preexisting CSF shunt, abnormal ICP, abnormal noninvasive imaging findings, and BMI $\geq$ 25 ) in relation to venographic results were used to obtain diagnostic accuracy, including true and false positive and negative, sensitivity, specificity, and positive and negative predictive value (PPV and NPV, respectively). Multivariate logistic regression was used to identify factors related to CVPG, and receiver operating characteristic analysis was also performed. Cluster analysis was performed to account for the patients who underwent multiple procedures. Statistical significance was defined as $\mathrm{p}<0.01$; IBM SPSS Statistics for Windows, version 22 (IBM Corp.) and MedCalc Statistical Software version 14.12.0 (MedCalc Software bvba) were used for statistical analyses.

\section{Results}

In total, 164 consecutive venograms were performed in 155 patients; there were no complications related to the procedure. Two patients underwent 3 venogram procedures and 5 patients underwent 2 venogram procedures, with all repeat procedures performed at least 3 months after the initial procedure. Cohort characteristics are shown in Table 1. Recent BMI measurements were available in 117 patients, the majority of whom $(78.6 \%[\mathrm{n}=92])$ were overweight (BMI $\geq 25)$ and more than half $(53.8 \%$ [n = 63]) of whom were obese (BMI $\geq 30)$ at the time of their procedure. The most common headache etiology in the 
TABLE 1. Cohort characteristics of 164 venogram procedures in 155 headache patients*

\begin{tabular}{lc}
\hline \multicolumn{1}{c}{ Characteristic } & Value \\
\hline Age (yrs) & $32.79 \pm 13.88$ \\
\hline Mean & $25(15 \%)$ \\
\hline Age $<18$ & $32.53 \pm 9.51$ \\
\hline BMI & $92(78.6 \%) \dagger$ \\
\hline Mean & $132(80.7 \%)$ \\
\hline BMI $\geq 25$ & \\
\hline Female sex & $96(58.5 \%)$ \\
\hline Headache diagnosis & $11(6.7 \%)$ \\
\hline Idiopathic intracranial hypertension & $10(6.1 \%)$ \\
\hline Congenital hydrocephalus & $8(4.9 \%)$ \\
\hline Chiari malformation & $5(3.0 \%)$ \\
\hline Extracranial jugular vein compression & $3(1.8 \%)$ \\
\hline Tumor or mass compressing sinus & $31(18.9 \%) \ddagger$ \\
\hline Postconcussive & $60(36.6 \%)$ \\
\hline Other & $66(85.7 \%) \S$ \\
\hline Preexisting shunt & $73(65.2 \%) \uparrow$ \\
\hline Abnormal ICP $\left(\geq 25\right.$ cm $\left.\mathrm{H}_{2} \mathrm{O}\right)$ & $42(25.6 \%)^{* *}$ \\
\hline Abnormal findings on noninvasive CV imaging
\end{tabular}

$\mathrm{CV}=$ cerebrovascular.

* Values represent numbers of procedures (\%) unless otherwise indicated. Multiple procedures performed in the same patient over time may show different results; however, cluster analysis did not show an effect of repeated measures. Data are thus based on number of procedures.

† Percentage is from 117 procedures with available and recent BMI measurements.

$\ddagger$ Includes migraines; chronic daily headaches; history of previous sinus thrombosis with persistent headaches despite recanalization, tumor resection, or aneurysm clipping.

$\S$ Percentage is from 77 procedures with preceding ICP measurement.

If Percentage is from 112 procedures with preceding noninvasive cerebrovascular imaging (MR or CT venography or angiography).

** Percentage is from 164 procedures.

155 patients was IIH $(61.9 \%$ [n = 96]). The "other" diagnosis category was composed of heterogeneous headache diagnoses that did not meet IIH criteria, including migraines, chronic daily headaches, history of previous sinus thrombosis with persistent headaches despite recanalization, and history of tumor resection or aneurysm clipping. Sixty (38.7\%) of 155 patients had preexisting CSF shunts. Seventy-seven (49.7\%) patients underwent ICP measurement within 4 weeks of venography, and $66(85.7 \%)$ of these 77 demonstrated elevated ICP. Noninvasive imaging had been performed within the 3 months preceding the procedure in 112 cases, and $73(65.2 \%)$ of these imaging studies showed dural venous sinus abnormalities such as stenosis or atresia.

The overall incidence of CVPG was $25.6 \%$ (present in 42 of 164 procedures). The average gradient was $22 \pm 16$ $\mathrm{mm} \mathrm{Hg}$ (range $8-81 \mathrm{~mm} \mathrm{Hg}$ ). CVPG was located between the transverse and sigmoid sinuses in all but 2 procedures (in one it was between the anterior and posterior one-third of the superior sagittal sinus and in another it was between the jugular bulb and the extracranial internal jugular vein). Twenty-eight procedures resulted in bilateral CVPG. The average superior sagittal sinus pressure in procedures with and without CVPG was $33.5 \mathrm{~mm} \mathrm{Hg}$ and $14.6 \mathrm{~mm} \mathrm{Hg}$, respectively $(\mathrm{p}<0.001)$.

CVPG was found in 34 of 96 procedures (35.4\%) in patients with IIH and in 8 of 68 procedures (11.8\%) in nonIIH patients; IIH was significantly associated with CVPG on univariate analysis $(\mathrm{p}<0.001)$. No patient with Chiari malformation, posttraumatic headache, or tumor with dural sinus compression had CVPG. Of the 8 procedures in non-IIH patients with CVPG, 2 procedures were in 2 patients (ages 2 and 15) with a diagnosis of congenital hydrocephalus, both of whom had ventriculoperitoneal shunts in place. The patient who was 15 years old at the time of the venogram had undergone a lumbar puncture that demonstrated elevated ICP. The 2-year-old patient had symptoms of intermittent shunt malfunction but had not undergone recent ICP measurement. The remaining 6 procedures in which CVPG was identified in the absence of IIH were in patients with the following headache diagnoses: remote history of vestibular schwannoma resection, remote history of meningitis, pulsatile tinnitus, extracranial styloid compression of the jugular vein, remote history of cerebral venous sinus thrombosis (which was radiographically resolved), and chronic headaches not otherwise specified.

CVPG was identified during only $8.3 \%(n=5)$ of the procedures in the 60 patients with a preexisting shunt, and in $0 \%$ of the 11 procedures in the 77 patients with normal ICP ( $p<0.001$ for both). There was a trend toward association between abnormalities on noninvasive imaging and CVPG $(\mathrm{p}=0.07)$.

Cross-tabulations of major patient characteristics at the time of the procedure (BMI $\geq 25$, preexisting shunt, abnormal ICP, abnormal findings on noninvasive cerebrovascular imaging) were performed for the entire sample and for procedures in patients with IIH (Table 2) to determine the predictive accuracy for CVPG. Importantly, abnormal ICP was associated with a $100 \%$ NPV, as CVPG was not observed in any procedure in patients with normal ICP. A non-overweight BMI $(<25)$ was also associated with a high NPV (71.4\% for procedures in patients with IIH and $88.0 \%$ overall). No factor was an efficient positive predictor of CVPG. Cluster analysis did not reveal any significant effect of the 7 patients who underwent a total of 9 additional procedures.

The results of multivariate and receiver operating characteristic analyses are shown in Table 3 and Fig. 1. Because of a substantial overlap between IIH and obesity, BMI was not included in the multivariate analysis. ICP was also excluded because no patient with normal ICP had CVPG, and the analysis was limited to 112 procedures in patients who had prior noninvasive imaging. The diagnosis of IIH was a significant positive predictor of CVPG (OR 4.97, 95\% CI 1.71-14.47), whereas CVPG was significantly less likely to be found during procedures in patients with preexisting shunts (OR 0.09, 95\% CI 0.02-0.44). Noninvasive imaging abnormality, age, and sex were not predictors of CVPG. The area under the curve for Fig. 1 was 0.79 (95\% CI 0.70-0.88). 


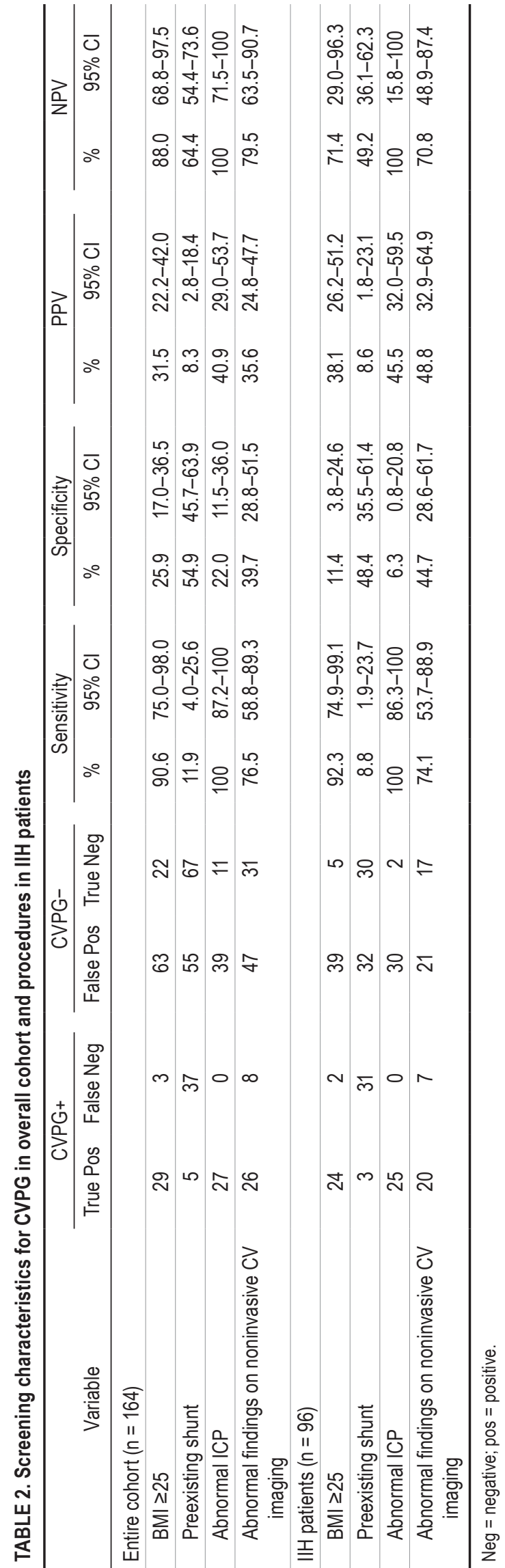

TABLE 3. Multivariate analysis of predictors of CVPG in procedures preceded by noninvasive cerebrovascular imaging $(n=112)$

\begin{tabular}{ccc}
\hline \multicolumn{1}{c}{ Variable } & Odds Ratio & $95 \% \mathrm{Cl}$ \\
\hline Age $(\mathrm{yrs})$ & 0.99 & $0.96-1.03$ \\
\hline Female sex & 0.93 & $0.25-3.49$ \\
\hline Diagnosis of IIH & 4.97 & $1.71-14.47^{*}$ \\
\hline Preexisting shunt & 0.09 & $0.02-0.44^{*}$ \\
\hline${ }^{*} p<0.01$. & &
\end{tabular}

\section{Discussion}

We have reported the overall incidence of CVPG in IIH and non-IIH patients in a large cohort of headache patients. A diagnosis of IIH was a significant positive predictor of CVPG. Normal ICP and previous CSF shunting were significant negative predictors, and noninvasive imaging results were not helpful for screening. Headache diagnoses such as Chiari malformation, postconcussive headache, and a tumor compressing the dural venous sinuses were not associated with CVPG, although the sample size for each of these diagnoses was small.

The relationship between dural venous sinus stenosis and headache, especially with respect to IIH, and the efficacy of venous stenting have been established in several series and meta-analyses. ${ }^{1,2,16,18,21}$ Authors have suggested both causative and reactive relationships between IIH, headache, and dural venous sinus stenosis, but the exact mechanism has not been elucidated. ${ }^{8,20}$ Although elevated ICP (and the attendant elevation in cerebral venous pressure) is thought to be a component of both IIH and secondary intracranial hypertension, ${ }^{13}$ not all patients with headache, elevated ICP, and elevated cerebral venous pressure demonstrate a CVPG across a focal anatomical location such as the transverse-sigmoid sinus junction. However, most endovascular operators use the presence of a CVPG to determine which patients would benefit from endovascular stenting of the affected dural venous sinus. ${ }^{1}$ Thus the overall rate of CVPG, as well as clinical risk factors for CVPG among IIH and non-IIH headache patients, would be useful for screening headache patients for possible endovascular intervention.

Elevated ICP is a hallmark of IIH that is often, but not always, present in other headache syndromes. ${ }^{12}$ While it is possible for elevated ICP to be the result of CVPG, we hypothesize that since no patient in our cohort with normal ICP demonstrated CVPG, elevated ICP is instead the result of dynamic resistance in relation to dural venous sinus stenosis. ${ }^{4}$ In this model, elevated ICP precipitates stenosis in certain patients with focal weakness in the dural sinus wall. The elevated cerebral venous pressure upstream from the stenosis prevents the bulk flow resorption of CSF, resulting in a positive feedback loop of increased ICP and further stenosis. Patients without elevated ICP, even in the setting of anatomically narrowed dural venous sinuses, either do not suffer from this positive feedback loop (with the stenosis constituting a normal anatomical variant without physiological consequences in these individuals) or have elevated pressure throughout the entire 


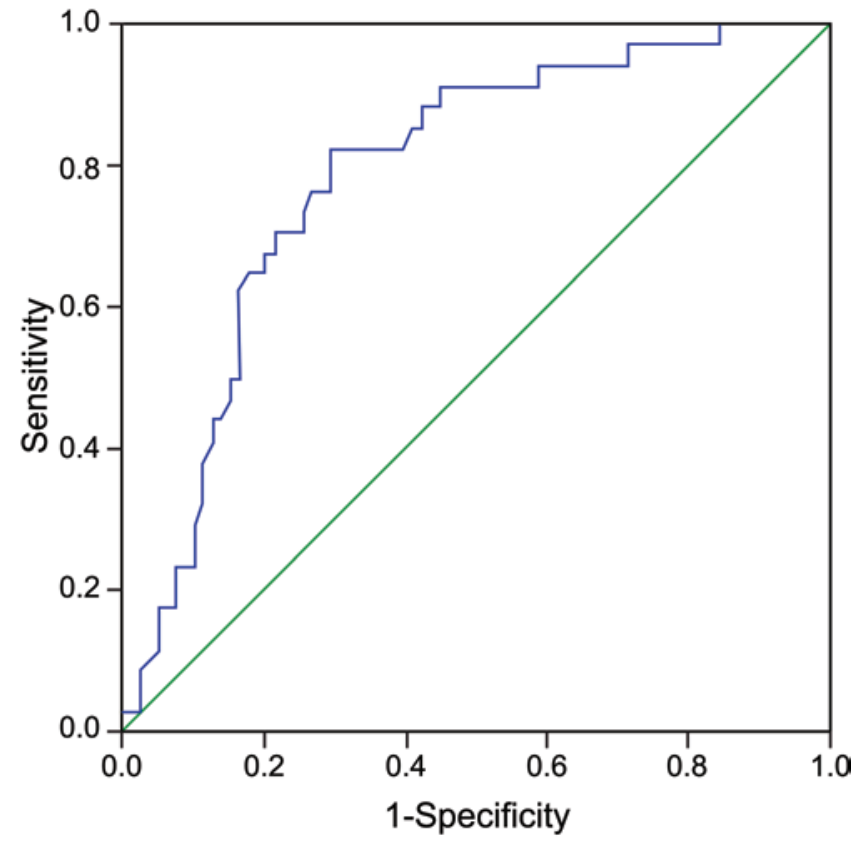

FIG. 1. Receiver operating characteristic graph of multivariate analysis (see Table 3 ) of predictors for CVPG. The area under the curve is 0.79 $(95 \% \mathrm{Cl} 0.70-0.88)$. Figure is available in color online only.

venous system (and thus no CVPG). ${ }^{17}$ Neither of these types of patients would benefit from focal stenting of the dural venous sinus stenosis, as the former does not have physiologically elevated cerebral venous pressure and the latter has diffusely elevated pressure for which the focal stenosis is not solely responsible. This may explain why patients with a preexisting shunt have a low likelihood of CVPG on both univariate and multivariate analysis, as ICP is likely to be within normal limits in such patients. Indeed, many patients in our cohort who had preexisting shunts presented with worsening headache and (in some cases) elevated ICP suggestive of shunt failure. Thus any patient with concern for headache related to elevated cerebral venous pressure should undergo lumbar puncture or shunt interrogation, and only those with elevated ICP should be screened for CVPG with cerebral venography and manometry.

Eight patients without diagnosed IIH had CVPG. It is unclear why the 2 patients with both congenital hydrocephalus and CVPG differed from the 12 other patients with congenital hydrocephalus who did not have CVPG, all of whom had shunts in place. Although pediatric IIH has been reported, ${ }^{10}$ both of our pediatric patients had ventriculomegaly on previous cranial imaging, suggesting another etiology that resulted in dural venous incompetence. Another patient with a remote history of extensive dural venous sinus thrombosis (since resolved) likely had secondary intracranial hypertension similar in etiology to $\mathrm{IIH}$, given her bilateral transverse sinus focal stenosis in the location of the resolved sinus thrombosis. Extracranial jugular venous compression has been reported as a distinct headache entity, ${ }^{7}$ and its relation to elevated cerebral venous pressure is evidenced by the patient with such compression and CVPG. A communicating hydrocephalus was likely undiagnosed in both the postmeningitic patient and the patient with previous jugular schwannoma resection. Finally, in the 2 remaining non-IIH patients with CVPG, presenting symptoms and the absence of neuroimaging findings strongly suggest undiagnosed $\mathrm{IIH}$, although no formal diagnosis was established because these patients were referred from other institutions specifically for venography. Thus patients with IIH-like symptoms may benefit from venography in rare cases, especially when they have elevated ICP.

Noninvasive imaging can reveal dural venous sinus abnormalities, although these may represent normal anatomical variants in up to $31 \%$ of cases. ${ }^{3}$ While there is a good correlation between headache and dural venous sinus abnormalities on noninvasive imaging such as CT venography or MR venography, our results dispute that such imaging should be used as a screening tool to determine whether patients should undergo invasive cerebral venography, as previously suggested. ${ }^{1}$ A study of 29 IIH patients and 59 controls demonstrated a correlation of $93 \%$ between IIH and dural venous sinus stenosis on MRV, compared with only $7 \%$ of asymptomatic controls. ${ }^{11}$ Similarly, an MRV study of 724 patients with migraine found bilateral transverse-sigmoid sinus stenosis in $6.7 \% .^{6}$ Another report of 217 patients who underwent both MRV and lumbar puncture for various neurological indications found that of the $11.1 \%$ of patients with elevated ICP, $100 \%$ had bilateral transverse-sigmoid sinus stenosis, while the rate of bilateral stenosis in those with normal ICP was $13.4 \% .^{5}$ These results are similar to those in another study in which $16 \%$ of 44 patients with venous sinus stenosis on MRV had normal ICP. ${ }^{14}$ However, it is unclear whether symptom severity is specifically related to bilateral (vs unilateral) stenosis. There is a significant association between Chiari malformation and transverse sinus stenosis on MRI ${ }^{19}$ although in our series no patient with Chiari malformation had CVPG.

Although previous studies suggest a correlation between noninvasive imaging results and $\mathrm{IIH}$, the rate of CVPG among patients with noninvasive imaging evidence of dural sinus stenosis has not been previously described. Our results suggest that noninvasive imaging may not accurately predict CVPG: the PPV was $<50 \%$, and multivariate analyses failed to demonstrate noninvasive imaging as a significant predictor of CVPG. Indeed, 8 (20.5\%) of 39 patients in our cohort with CVPG had anatomically normal venous sinuses on noninvasive imaging. Conversely, patients with normal anatomical variants as well as those with mass lesions extrinsically compressing a dural venous sinus may have alternative or compensatory venous drainage without an elevation in cerebral venous pressures, as was seen in the 5 patients in our cohort with tumors causing compressive venous sinus stenosis, none of whom demonstrated any elevation in venous sinus pressure or CVPG. There was no significant correlation between bilateral stenosis on noninvasive imaging and bilateral CVPG, although our sample size was too small (n $=28$ ) to completely rule out such an association. Importantly, there was a trend $(\mathrm{p}=0.07)$ toward a univariate association between abnormalities on noninvasive imaging 
and CVPG. Nevertheless, only $68.3 \%$ of the procedures in this study were preceded by noninvasive imaging, so our study may be underpowered to detect a robust association. It remains to be seen whether screening patients as candidates for venography and possible stenting on the basis of noninvasive cerebrovascular imaging results is beneficial.

Given our findings, our approach to venography and manometry for headache patients can be summarized as follows. In patients with headache in whom IIH or elevated ICP is suspected, lumbar puncture should be performed. In those patients with elevated ICP without a preexisting CSF shunt, cerebral venography and manometry should be performed to rule out CVPG. Routine noninvasive cerebrovascular imaging may not be predictive of CVPG (although cranial imaging is still recommended to rule out secondary causes of intracranial hypertension), so we do not routinely perform CTV or MRV prior to venography, although such imaging may be shown in future studies to improve patient selection. A high index of suspicion should be in place for IIH patients in particular, whereas patients with normal ICP should not undergo venography unless other compelling clinical factors are present (such as a severe visual field deficit).

This study has several limitations. Its retrospective nature introduces a selection bias toward patients with a diagnosis of IIH. The overall incidence of CVPG in the non-IIH population should be interpreted with caution, as non-IIH referrals were at the discretion of the referring physician, and quantification of headache severity was not routinely performed. However, venographic results from non-IIH headache patients have not been previously described, and a proportion of non-IIH patients demonstrated CVPG, which may expand the indications for venography. Indeed, the incidence of CVPG in this study may underestimate the rate in headache patients who do not have IIH or a CSF shunt, because $34.8 \%$ (23 of 66) of our non-IIH headache patients had a preexisting shunt. Finally, incomplete availability of patient characteristics such as ICP measurement and noninvasive cerebrovascular imaging results weakens the power of our statistical analysis and limits the generalizability of our findings. A future study with prospective standardized data collection of these variables is required to refine and validate our preliminary findings.

\section{Conclusions}

CVPG was present in $35.4 \%(n=34)$ of the 96 procedures conducted in $96 \mathrm{IIH}$ patients. Patients with normal ICP did not demonstrate CVPG, and the presence of a preexisting shunt significantly reduced its likelihood. Patients with elevated ICP (especially with IIH) without previous shunting should be routinely considered for venography and manometry to rule out CVPG.

\section{Acknowledgments}

We thank Kristina Chapple, $\mathrm{PhD}$, for her assistance with statistical analysis.

\section{References}

1. Ahmed RM, Wilkinson M, Parker GD, Thurtell MJ, Macdonald J, McCluskey PJ, et al: Transverse sinus stenting for idiopathic intracranial hypertension: a review of 52 patients and of model predictions. AJNR Am J Neuroradiol 32:1408-1414, 2011

2. Albuquerque FC, Dashti SR, Hu YC, Newman CB, Teleb M, McDougall CG, et al: Intracranial venous sinus stenting for benign intracranial hypertension: clinical indications, technique, and preliminary results. World Neurosurg 75:648652, 592-595, 2011

3. Ayanzen RH, Bird CR, Keller PJ, McCully FJ, Theobald MR, Heiserman JE: Cerebral MR venography: normal anatomy and potential diagnostic pitfalls. AJNR Am J Neuroradiol 21:74-78, 2000

4. Bateman GA: Arterial inflow and venous outflow in idiopathic intracranial hypertension associated with venous outflow stenoses. J Clin Neurosci 15:402-408, 2008

5. Bono F, Cristiano D, Mastrandrea C, Latorre V, D'Asero S, Salvino D, et al: The upper limit of normal CSF opening pressure is related to bilateral transverse sinus stenosis in headache sufferers. Cephalalgia 30:145-151, 2010

6. Bono F, Messina D, Giliberto C, Cristiano D, Broussard G, Fera F, et al: Bilateral transverse sinus stenosis predicts IIH without papilledema in patients with migraine. Neurology 67:419-423, 2006

7. Dashti SR, Nakaji P, Hu YC, Frei DF, Abla AA, Yao T, et al: Styloidogenic jugular venous compression syndrome: diagnosis and treatment: case report. Neurosurgery 70:E795-E799, 2012

8. De Simone R, Ranieri A, Bonavita V: Advancement in idiopathic intracranial hypertension pathogenesis: focus on sinus venous stenosis. Neurol Sci 31 (Suppl 1):S33-S39, 2010

9. De Simone R, Ranieri A, Montella S, Erro R, Fiorillo C, Bonavita V: Sinus venous stenosis-associated IIHWOP is a powerful risk factor for progression and refractoriness of pain in primary headache patients: a review of supporting evidences. Neurol Sci 32 (Suppl 1):S169-S171, 2011

10. Dwyer CM, Prelog K, Owler BK: The role of venous sinus outflow obstruction in pediatric idiopathic intracranial hypertension. J Neurosurg Pediatr 11:144-149, 2013

11. Farb RI, Vanek I, Scott JN, Mikulis DJ, Willinsky RA, Tomlinson G, et al: Idiopathic intracranial hypertension: the prevalence and morphology of sinovenous stenosis. Neurology 60:1418-1424, 2003

12. Friedman DI, Jacobson DM: Diagnostic criteria for idiopathic intracranial hypertension. Neurology 59:1492-1495, 2002

13. Karahalios DG, Rekate HL, Khayata MH, Apostolides PJ: Elevated intracranial venous pressure as a universal mechanism in pseudotumor cerebri of varying etiologies. Neurology 46:198-202, 1996

14. Kelly LP, Saindane AM, Bruce BB, Ridha MA, Riggeal BD, Newman NJ, et al: Does bilateral transverse cerebral venous sinus stenosis exist in patients without increased intracranial pressure? Clin Neurol Neurosurg 115:1215-1219, 2013

15. Lai LT, Danesh-Meyer HV, Kaye AH: Visual outcomes and headache following interventions for idiopathic intracranial hypertension. J Clin Neurosci 21:1670-1678, 2014

16. Puffer RC, Mustafa W, Lanzino G: Venous sinus stenting for idiopathic intracranial hypertension: a review of the literature. J Neurointerv Surg 5:483-486, 2013

17. Rohr A, Bindeballe J, Riedel C, van Baalen A, Bartsch T, Doerner L, et al: The entire dural sinus tree is compressed in patients with idiopathic intracranial hypertension: a longitudinal, volumetric magnetic resonance imaging study. Neuroradiology 54:25-33, 2012

18. Goodwin CR, Elder BD, Ward A, Orkoulas-Razis D, Kosztowski TA, Hoffberger J, et al: Risk factors for failed transverse sinus stenting in pseudotumor cerebri patients. Clin Neurol Neurosurg 127:75-78, 2014

19. Saindane AM, Bruce BB, Desai NK, Roller LA, Newman NJ, Biousse V: Transverse sinus stenosis in adult patients 
with Chiari malformation type I. AJR Am J Roentgenol 203:890-896, 2014

20. Stevens SA, Previte M, Lakin WD, Thakore NJ, Penar PL, Hamschin B: Idiopathic intracranial hypertension and transverse sinus stenosis: a modelling study. Math Med Biol 24:85-109, 2007

21. Teleb MS, Cziep ME, Lazzaro MA, Gheith A, Asif K, Remler B, et al: Idiopathic intracranial hypertension. A systematic analysis of transverse sinus stenting. Interv Neurol 2:132143,2013

\section{Disclosures}

The authors report no conflict of interest concerning the materials or methods used in this study or the findings specified in this paper.

\section{Author Contributions}

Conception and design: Albuquerque, Levitt. Acquisition of data: Albuquerque, Levitt, Hlubek, Moon, Kalani, McDougall. Analysis and interpretation of data: Albuquerque, Levitt, Hlubek. Drafting the article: Albuquerque, Levitt. Critically revising the article: all authors. Reviewed submitted version of manuscript: all authors. Statistical analysis: Albuquerque, Levitt. Administrative/ technical/material support: Albuquerque, Levitt, Hlubek, Moon. Study supervision: Albuquerque.

\section{Correspondence}

Felipe C. Albuquerque, c/o Neuroscience Publications, Barrow Neurological Institute, St. Joseph's Hospital and Medical Center, 350 W. Thomas Rd., Phoenix, AZ 85013. email: neuropub@ dignityhealth.org. 